\title{
Seasonal study of contamination by metal in water and sediment in a sub-basin in the Southeast of Brazil
}

\author{
Chiba, WAC. ${ }^{a *}$, Passerini, MD. ${ }^{a, b}$, Baio, JAF. ${ }^{c}$, Torres, JC. ${ }^{b}$ and Tundisi, JG. ${ }^{a, b}$ \\ apprograma de Pós-Graduação em Ecologia e Recursos Naturais, Universidade Federal de São Carlos- UFSCar, \\ CP 676, CEP 13565-905, São Carlos, SP, Brazil \\ bInstituto Internacional de Ecologia, Rua Bento Carlos, 750, CEP 13560-660, São Carlos, SP, Brazil \\ 'Instituto de Química, Universidade de São Paulo - USP, Av. Trabalhador São-Carlense, 400, \\ CEP 13560-970, São Carlos, SP, Brazil \\ *e-mail: wagner.chiba@ hotmail.com
}

Received June 29, 2010 - Accepted October 21, 2010 - Distributed November 30, 2011

(With 4 figures)

\begin{abstract}
The spatial and temporal occurrence of heavy metals ( $\mathrm{Al}, \mathrm{Cd}, \mathrm{Pb}, \mathrm{Zn}, \mathrm{Cr}, \mathrm{Co}, \mathrm{Cu}, \mathrm{Fe}, \mathrm{Mn}$ and $\mathrm{Ni}$ ) in water and sediment samples was investigated in a sub-basin in the southeast of Brazil (São Carlos, SP). All samples were analysed using the USEPA adapted metal method and processed in an atomic absorption spectrophotometer. The discriminant analysis demonstrated that there are significant seasonal differences of metal distribution in the water data, but there are no differences to sediment. The basin studied has high levels of contamination by toxic metals in superficial water and sediment. The superficial water, in the rainy season, presented high levels of $\mathrm{Cr}, \mathrm{Ni}, \mathrm{Pb}$ and $\mathrm{Cd}$, while in the dry season it presented high levels of $\mathrm{Zn}$ and Ni. The Principal Component Analysis demonstrated that the season has a huge influence on the levels, types and distribution of metals found in water. The source of contamination was probably diffuse, due to products such as batteries and fluorescent lamps, whose dump discharge can contaminate the bodies of water in the region in the rainy season. Due to fires from the harvest of sugar cane, high levels of $\mathrm{Zn}$ were found into the environment, in the dry season.
\end{abstract}

Keywords: heavy metal, water pollution, sediment pollution.

\section{Estudo sazonal da contaminação por metais na água e sedimentos em uma sub-bacia na região sudeste do Brasil}

\begin{abstract}
Resumo
A ocorrência espacial e temporal de metais pesados ( $\mathrm{Al}, \mathrm{Cd}, \mathrm{Pb}, \mathrm{Zn}, \mathrm{Cr}, \mathrm{Co}, \mathrm{Cu}, \mathrm{Fe}, \mathrm{Mn}$ e $\mathrm{Ni}$ ) nas amostras de água e sedimento foram investigadas em uma sub-bacia do sudeste do Brasil (São Carlos, SP). Todas as amostras foram analisadas através do método de metal USEPA, adaptado e processado por espectrofotômetro de absorção atômica. A análise de Discriminante demonstrou que existem diferenças sazonais significantes na distribuição dos metais na água, mas não existem diferenças para o sedimento. A água superficial, na estação chuvosa, apresentou altos índices de $\mathrm{Cr}, \mathrm{Ni}$, $\mathrm{Pb}$ e Cd, enquanto na estação seca apresentou altos índices de Zn e Ni. A Análise de Componente Principal demonstrou que as estações do ano têm uma enorme influência no nível, no tipo e na distribuição dos metais encontrados na água. A fonte de contaminação foi provavelmente difusa devido a produtos como baterias e lâmpadas fluorescentes, cuja descarga nos lixões pode contaminar os corpos de água da região na estação chuvosa. Devido a queimadas provenientes da colheita de cana-de-açúcar, altos níveis de $\mathrm{Zn}$ foram encontrados no ambiente na estação de seca.
\end{abstract}

Palavras-chave: metal pesado, poluição da água, poluição do sedimento. 


\section{Introduction}

Water is a vital element to living organisms and its inadequate use for different purposes has caused several problems both to quality and amount of this resource. Also, due to exponential increase in water demand and water quality deterioration because of lack of domestic and industrial effluent treatment, water started to be considered a limited resource, being the main cause for concern in different scales, from local to global (Tundisi, 2003).

Pollutants, when introduced to an aquatic system, present a heterogeneous behaviour, and they can be toxic just by their presence or by degradation process. Pollutants can release compounds, which assimilated by organisms, can interfere in physiologic processes, such as reproductive aspects, survival and, consequently, changing the population structure (Boudou and Ribeyre, 1989; Doi et al., 2007). Although adverse health effects of heavy metals have been known for a long time, exposure to heavy metals continues and is even increasing in some areas (Järup, 2003).

Metals in natural water can play an important role in the biological functioning of many organisms. Some metals, depending on the way they are distributed in water, can present a high toxicity level to living organisms, while others are considered essential, such as Fe, Al, Zn. However, even essential metal can be toxic if in large concentration (Templeton et al., 2000).

Industrial activities refine metallic minerals, use metals as raw material for their products or dispose sub-products in their processes. Foundries, electroplating, automobile and paper industry, use and discard high amount of metals such as cadmium, lead, copper, nickel and zinc (PeláezRodríguez, 2001; Järup, 2003). Pesticides and fertilisers used in agricultural areas have large amounts of $\mathrm{Cu}$. Fires from the harvest of sugar cane release high levels of $\mathrm{Zn}$ into the environment (Ziolli, 1995). The deposit of household, industrial and health care waste, without a prior geological and hydrological study, emphasizes the possibility of soil, aquifers and surface water contamination.

According to IPT (1995), solid waste disposal can be done in three ways: in dumps (simply dumping of waste on the ground), at controlled landfills (disposal of waste on the ground with a degree of control, compression and coating) and landfills (waste placement associated with engineering works, independently and safely in the least space as possible). Velozo (2006) showed environmental pollution in the city of São Carlos because of the presence of a closed dump, located in the basin of the Feijão river.

The characteristics of rivers vary not only according to the basin in which they are located. These features are consequences of the geology that makes up basin drainage, slope, vegetation, rainwater characteristics, various types of human activities, in addition to their variation over time and space (Goldman and Horne, 1983; Pedroso et al., 1988). This fact requires that quality assessments of water bodies should be made in the course of a year, with periods of drought and rainfall. Thus, the analysis of variables on water quality, associated with indicators of chemical and the study of the area in an integrated way, provide relevant information for the spatial characterisation of bodies of water in river basins (Goldman and Horne, 1983).

According to Boyd (2010) there is the need to explore ecosystem studies on metals in larger-scale than toxicological studies. Despite being more difficult to implement, the study involving the collection of information about presence, distribution, quality and quantity of contaminants in different matrices such as water and sediment, provides useful information for assessing environmental risks and therefore for ecological monitoring and human health (Zimmer and Zimmer, 2008).

In recent years, many studies have been conducted on the evaluation of metals in freshwater environments (Sanei et al., 2010; Mdegela et al., 2009; Kikuchi et al., 2009; Brahimpour and Mushrifah, 2008; Mastoi et al., 2008). However, there are few studies involving the influence of seasonality as a key factor in determining the sources of pollutants.

In the region of São Carlos, some studies were conducted to evaluate the environmental conditions of lotic bodies, as Rios (1993), Santos (1993) and Teixeira (1993). Specifically for the Monjolinho river basin, several studies have been carried out on various aspects, including those developed by Povinelli (1972), Gomes (1981), Santos (1990), Sé (1992), Salami (1996) and Pelaez-Rodriguez (2001). Only Pelaez-Rodriguez (2001) focused on the analysis of metals in bodies of water of the Jacaré-Guaçú basin.

Given the importance of studying metals as pollutants of aquatic ecosystems and the absence of behavioural studies of this pollutant, considering the watershed as a whole, from the southeast of Brazil, this project aimed to evaluate the concentration of metal in the basins of Monjolinho and Feijão rivers. The study aimed to evaluate the concentrations of the metals $\mathrm{Al}, \mathrm{Cd}, \mathrm{Pb}, \mathrm{Zn}, \mathrm{Cr}, \mathrm{Co}$, $\mathrm{Cu}, \mathrm{Fe}, \mathrm{Mn}$ and $\mathrm{Ni}$ in the water and sediment, to quantify and investigate the routes of intake of these metals for the watershed.

\section{Methods}

\subsection{Study area}

The city of São Carlos is located in central São Paulo State, between coordinates $47^{\circ} 30^{\prime}$ and $48^{\circ} 30^{\prime}$ 'Longitude, $21^{\circ} 30^{\prime}$ and $22^{\circ} 30^{\prime}$ Latitude. The area of the city is $1.132 \mathrm{~km}^{2}$, with a population of about 219,000 inhabitants, of whom $93.6 \%$ are of an urban settlement. The climate is classified as humid subtropical (Tolentino, 1967) or mild mesothermal (Nimer, 1972), with hot and humid summer from October to March, and cold and dry winter from April to September. The draining of the city has a dendritic pattern, reflecting the regional geology. The city has a rich water supply and the Monjolinho river is responsible for major urban drainage, crossing the city from east to west. The streams of Gregório, St. Maria Madalena and Tijuco Preto, which are Monjolinho tributaries, are located in 
the centre and north of São Carlos. They all have small flow and stabilised profiles. The Feijão river, the main surface water source that supplies the city of São Carlos, is $13 \mathrm{~km}$ from the centre of the city. The other surface water catchment is the Espraiado, near Monjolinho river. Figure 1 shows the sampling sites and Table 1 shows the rivers and corresponding sampling sites.

\subsubsection{Sampling methodology and procedures}

Samples were collected in February and March 2008 (rainfall) and September 2008 (dry) in twenty-six sites of the city of São Carlos (SP, Brazil), in urban, agricultural and natural areas, for water and sediment. The surface water samples were collected in $500 \mathrm{~mL}$ polyethylene gallon and kept away from the sun and heat in styrofoam boxes with ice for laboratory analysis. The collected water was filtered through a glass fiber filter Whatman GF/C with a porosity of $1.2 \mathrm{~mm}$. The samples were preserved in polyethylene bottles of $150 \mathrm{~mL}$ with $2 \mathrm{~mL}$ of $\mathrm{HNO}_{3}$ and then frozen (APHA, 2005).

\subsubsection{Sediment methodology}

The sediment was collected with the aid of plastic spatulas and stored at $4{ }^{\circ} \mathrm{C}$. The content of metals in sediment samples was assessed according to USEPA 3050B.
A reference material from Ultra Scientific - Catalog IRM008 and lot J408 - was used to check if the methodology used was appropriate for determination of metals in the sediment. Five tests were performed using standard synthetic sediment, which proved the effectiveness of the method.

Table 1. Rivers and corresponding sampling sites.

\begin{tabular}{cccc}
\hline Sites & $\begin{array}{c}\text { Water } \\
\text { bodies }\end{array}$ & Sites & Water bodies \\
\hline A1 & $\begin{array}{c}\text { Monjolinho } \\
\text { river }\end{array}$ & A12 & Espraiado stream \\
A8 & & A14 & \\
A9 & & A11 & Sta. Maria Madalena \\
A10 & & A15 & stream \\
A19 & & A16 & Tijuco Preto stream \\
A20 & & A17 & \\
A21 & & A18 & \\
A22 & & A23 & Água Quente stream \\
A2 & Gregório & A24 & \\
A3 & stream & A25 & Laranja Azeda stream \\
A4 & & & \\
A5 & & A26 & Feijão river \\
A6 & & & \\
A7 & & & \\
\hline
\end{tabular}
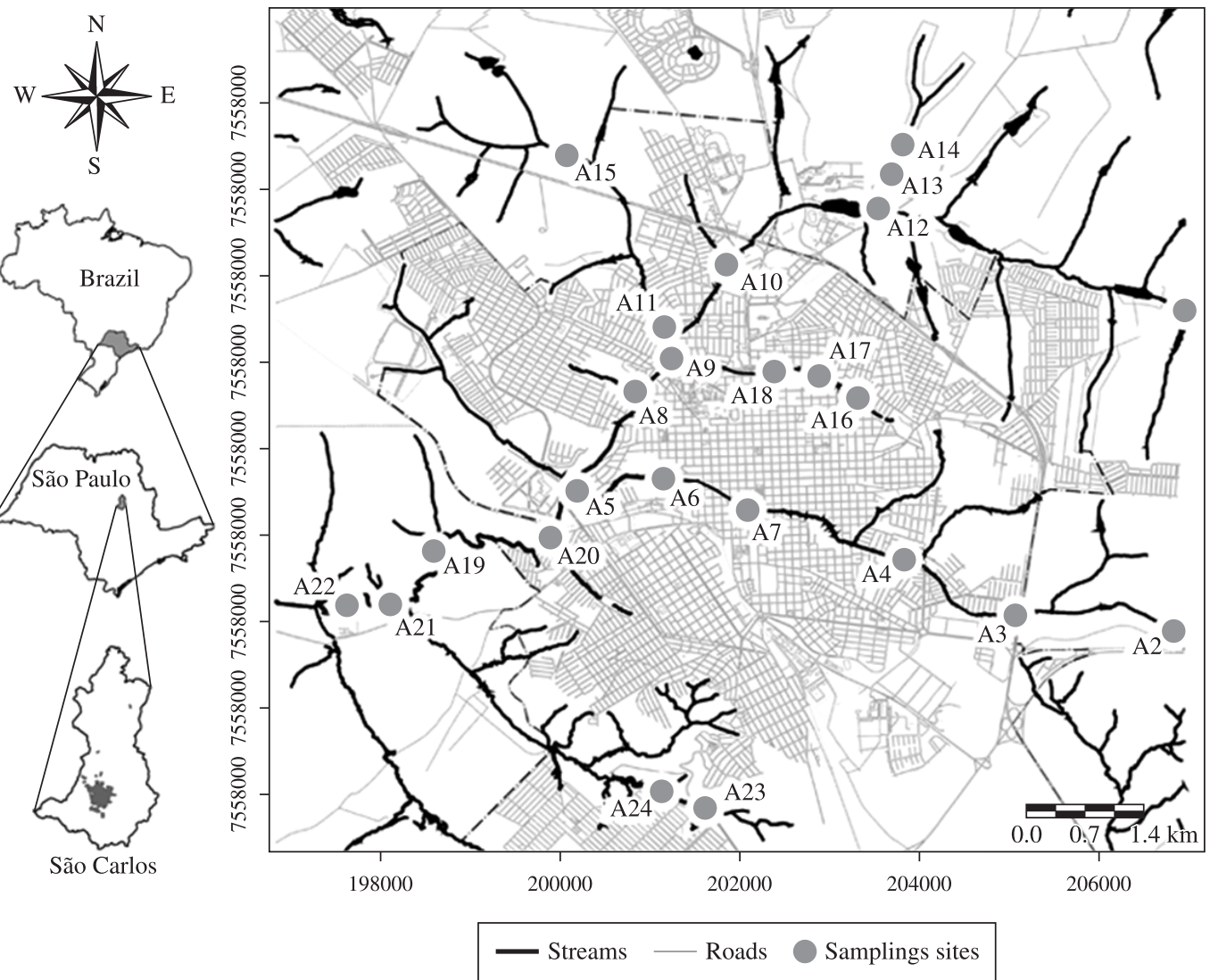

Figure 1. Study area: city of São Carlos. Sites A25 and A26, not represented, are located southeast of the city. 


\subsubsection{Superficial water methodology}

Physic chemistry parameters $(\mathrm{pH}$, conductivity, turbidity, dissolved oxygen, temperature, TDS and ORP), were verified with a Horiba multiparameter probe. All products collected were analysed with an atomic absorption spectrophotometer Varian, AA240 FS (Fast Sequential). The blanks were treated in the same way as the samples, ie, they received the reagents and they were read in triplicate. The detection limits of the analyses of metals were calculated according to INMETRO (2009).

\subsubsection{Climatological data}

Data on monthly rainfall compared with the climatological normal (1961-1990) were obtained for the city of São Carlos from the Instituto Nacional de Meteorologia (INMET, 2008) (Figure 2).

\subsubsection{Statistical analysis}

The values of metals concentration from water and sediment were normalised. There were two Discriminant analyses to verify if there were significant differences between the data from the rainy season and dry season for water and sediment. To analyse the spatial and temporal distribution of metals in the water, Principal Components Analysis (PCA) was performed. The PCA and the Discriminant analyses were performed using the software PAST v.1.95.

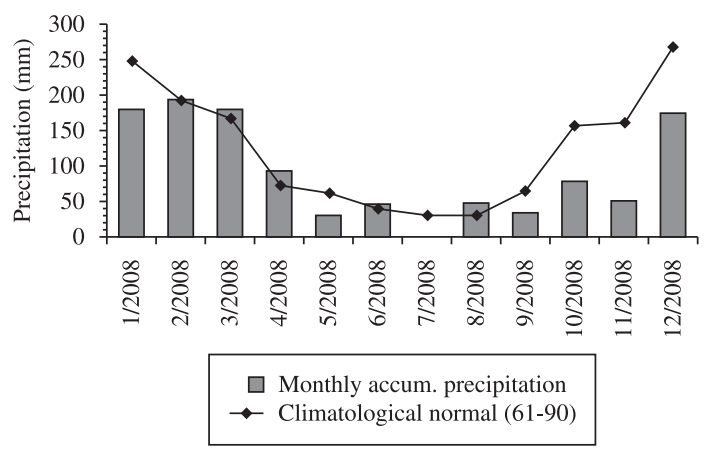

Figure 2. Accumulated precipitation x monthly climatological normal (1961-1990) for the municipality of São Carlos - SP, 2008. Source: INMET.

\section{Results}

\subsection{Chemical water parameters}

Table 2 presents the means, standard deviations and maximum and minimum values of chemical parameters of water conducted at sites for $\mathrm{pH}$, conductivity $(\mathrm{mS})$, dissolved oxygen (DO) (mg. $\left.\mathrm{L}^{-1}\right)$ and redox potential (ORP) $(\mathrm{mV})$.

\subsection{Metals procedures}

Table 3 presents the blank values and the detection limits of metal analysis in water and sediment.

\subsection{Metals in surface water: rainy season}

Metals found in water samples from São Carlos streams, in the collection conducted during the rainy season, are shown in Table 4, along with the limits established by Brazilian law for metals in water (Lgs. CONAMA 357/05, 2005).

It was found that the sites A19, A20, A21, A22, A23, A24, A25 and A26, have concentrations of cadmium, lead, copper and nickel above the permitted level for Class 3 waters and concentrations of cobalt and iron above the permitted level for Class 1 waters.

\subsection{Metals in surface water: dry season}

Metals found in water samples from streams in São Carlos, during the gathering held in the dry season, are shown in Table 5, together with the limits established by Brazilian law for metals in water (Lgs. CONAMA 357/05, 2005).

Cd values were above those provided for Class 1 waters in almost all sampling sites; the sites A21, A22, A23 and A24 presented the highest concentrations.

$\mathrm{Pb}$ presented values above the limits for Class 1 waters in A2, A4, A6, A13, A15, A16, A17, A18, A19, A20, A21, $\mathrm{A} 22, \mathrm{~A} 25$ and $\mathrm{A} 26$.

Levels of iron above the limit for Class 1 were found in most sites; its highest concentration was found in A1.

Most of the sites presented above Ni level permitted for Class 3 waters, reaching their highest values in the sites A17, A19 and A21 and A22.

Copper levels were higher than those provided for Class 3 CONAMA in A6, A7 and A19.

\subsection{Metals in sediment: the rainy and dry season}

Metal concentrations found in sediment samples from São Carlos streams, in rainy and drought periods, can be

Table 2. Chemical parameters of water: average, maximum and minimum value in the two collection periods, rain and drought.

\begin{tabular}{|c|c|c|c|c|c|c|}
\hline \multirow[b]{2}{*}{ Parameter } & \multicolumn{3}{|c|}{ Dry period } & \multicolumn{3}{|c|}{ Rainfall period } \\
\hline & Average & $\begin{array}{c}\text { Minimum } \\
\text { value }\end{array}$ & $\begin{array}{c}\text { Maximum } \\
\text { value }\end{array}$ & Average & $\begin{array}{c}\text { Minimum } \\
\text { value }\end{array}$ & $\begin{array}{c}\text { Maximum } \\
\text { value }\end{array}$ \\
\hline $\mathrm{pH}$ & 6.89 & 6.09 & 7.54 & 7.09 & 6.15 & 7.65 \\
\hline Condutivity (mS) & 0.12 & 0.01 & 0.47 & 0.17 & 0 & 0.715 \\
\hline OD $\left(m g . L^{-1}\right)$ & 8.27 & 7.2 & 9.6 & 5 & 0.73 & 9.77 \\
\hline ORP (mV) & 163.16 & 14 & 380 & 94.23 & 12 & 250 \\
\hline
\end{tabular}


Table 3. Blank values and deviation limits for water and sediment for the metal analysis

\begin{tabular}{|c|c|c|c|c|c|c|c|c|c|c|}
\hline \multicolumn{11}{|c|}{ Water $\left(\mathbf{m g} . \mathrm{L}^{-1}\right)$} \\
\hline & Al & Cd & $\mathbf{P b}$ & Zn & $\mathrm{Cr}$ & Co & $\mathrm{Cu}$ & $\mathrm{Fe}$ & Mn & $\mathrm{Ni}$ \\
\hline BV1w & -0.188 & 0.013 & 0.038 & 0.011 & 0.013 & 0.06 & 0.011 & 0.003 & 0.002 & 0.026 \\
\hline $\mathrm{BV} 2 \mathrm{w}$ & -0.175 & 0.013 & 0.038 & 0.009 & 0.017 & 0.066 & 0.009 & 0.001 & 0.003 & 0.026 \\
\hline $\mathrm{BV} 3 \mathrm{w}$ & -0.184 & 0.014 & 0.034 & 0.008 & 0.024 & 0.062 & 0.011 & 0.008 & 0.004 & 0.025 \\
\hline DL (mg. $\left.\mathrm{L}^{-1}\right)$ & 0.02 & 0.001 & 0.007 & 0.004 & 0.018 & 0.009 & 0.004 & 0.01 & 0.002 & 0.003 \\
\hline \multicolumn{11}{|c|}{ Sediment (mg.L $\left.{ }^{-1}\right)$} \\
\hline BV1s & 0.169 & -0.001 & 0.106 & 0.014 & 0.006 & 0.007 & 0.001 & 0.174 & 0.002 & 0.017 \\
\hline $\mathrm{BV} 2 \mathrm{~s}$ & 0.173 & -0.002 & 0.081 & 0.012 & 0.002 & 0.002 & 0.000 & 0.203 & 0.002 & 0.03 \\
\hline $\mathrm{BV} 3 \mathrm{~s}$ & 0.145 & -0.002 & 0.096 & 0.013 & 0.003 & 0.013 & 0.000 & 0.179 & -0.012 & 0.039 \\
\hline BV4s & 0.151 & -0.001 & 0.090 & 0.011 & 0.004 & 0.008 & 0.001 & 0.185 & -0.02 & 0.033 \\
\hline BV5s & 0.168 & -0.002 & 0.080 & 0.013 & 0.000 & 0.017 & 0.001 & 0.243 & 0.002 & 0.029 \\
\hline BV6s & 0.161 & -0.002 & 0.054 & 0.013 & 0.001 & 0.006 & 0.000 & 0.195 & 0.056 & 0.036 \\
\hline DL (mg.kg $\left.{ }^{-1}\right)$ & 3.29 & 0.17 & 5.34 & 0.30 & 0.59 & 1.61 & 0.15 & 7.51 & 7.95 & 2.35 \\
\hline
\end{tabular}

Table 4. Concentration of metals in the water during the rainy season $\left(\mathrm{mg} \cdot \mathrm{L}^{-1}\right)$.

\begin{tabular}{|c|c|c|c|c|c|c|c|c|c|c|}
\hline & Al & $\mathrm{Cd}$ & $\mathbf{P b}$ & $\mathrm{Zn}$ & $\mathrm{Cr}$ & Co & $\mathrm{Cu}$ & $\mathrm{Fe}$ & Mn & $\mathrm{Ni}$ \\
\hline A1 & $>\mathrm{DL}$ & $>\mathrm{DL}$ & $>\mathrm{DL}$ & 0.007 & $>\mathrm{DL}$ & $>\mathrm{DL}$ & $>\mathrm{DL}$ & $>\mathrm{DL}$ & 0.004 & $>\mathrm{DL}$ \\
\hline $\mathrm{A} 2$ & $>\mathrm{DL}$ & $>\mathrm{DL}$ & $>\mathrm{DL}$ & 0.009 & 0.018 & $>\mathrm{DL}$ & $>\mathrm{DL}$ & $>\mathrm{DL}$ & 0.023 & $>\mathrm{DL}$ \\
\hline A3 & $>\mathrm{DL}$ & $>\mathrm{DL}$ & $>\mathrm{DL}$ & 0.012 & $>\mathrm{DL}$ & $>\mathrm{DL}$ & 0.004 & 0.287 & 0.034 & $>\mathrm{DL}$ \\
\hline A4 & $>\mathrm{DL}$ & $>\mathrm{DL}$ & $>\mathrm{DL}$ & 0.009 & 0.049 & $>\mathrm{DL}$ & 0.006 & 0.218 & 0.023 & $>\mathrm{DL}$ \\
\hline A5 & $>\mathrm{DL}$ & $>\mathrm{DL}$ & $>\mathrm{DL}$ & 0.008 & $>\mathrm{DL}$ & $>\mathrm{DL}$ & 0.005 & 0.094 & 0.014 & $>\mathrm{DL}$ \\
\hline A6 & $>\mathrm{DL}$ & $>\mathrm{DL}$ & $>\mathrm{DL}$ & 0.034 & 0.021 & $>\mathrm{DL}$ & 0.006 & 0.280 & 0.021 & $>\mathrm{DL}$ \\
\hline A7 & $>\mathrm{DL}$ & $>\mathrm{DL}$ & $>\mathrm{DL}$ & $>\mathrm{DL}$ & 0.020 & $>\mathrm{DL}$ & 0.006 & 0.239 & 0.023 & $>\mathrm{DL}$ \\
\hline A8 & $>\mathrm{DL}$ & $>\mathrm{DL}$ & $>\mathrm{DL}$ & $>\mathrm{DL}$ & $>\mathrm{DL}$ & $>\mathrm{DL}$ & $>\mathrm{DL}$ & 0.068 & 0.010 & $>\mathrm{DL}$ \\
\hline A9 & $>\mathrm{DL}$ & $>\mathrm{DL}$ & $>\mathrm{DL}$ & $>\mathrm{DL}$ & 0.018 & $>\mathrm{DL}$ & 0.005 & 0.048 & 0.008 & $>\mathrm{DL}$ \\
\hline A10 & $>\mathrm{DL}$ & $>\mathrm{DL}$ & $>\mathrm{DL}$ & 0.013 & $>\mathrm{DL}$ & $>\mathrm{DL}$ & 0.007 & 0.059 & 0.007 & $>\mathrm{DL}$ \\
\hline A11 & $>\mathrm{DL}$ & $>\mathrm{DL}$ & $>\mathrm{DL}$ & 0.034 & $>\mathrm{DL}$ & $>\mathrm{DL}$ & 0.006 & 0.026 & 0.008 & $>\mathrm{DL}$ \\
\hline A12 & $>\mathrm{DL}$ & $>\mathrm{DL}$ & $>\mathrm{DL}$ & $>\mathrm{DL}$ & $>\mathrm{DL}$ & $>\mathrm{DL}$ & 0.004 & 0.023 & 0.013 & $>\mathrm{DL}$ \\
\hline A13 & $>\mathrm{DL}$ & $>\mathrm{DL}$ & $>\mathrm{DL}$ & $>\mathrm{DL}$ & $>\mathrm{DL}$ & $>\mathrm{DL}$ & 0.006 & 0.024 & $>\mathrm{DL}$ & $>\mathrm{DL}$ \\
\hline A14 & $>\mathrm{DL}$ & $>\mathrm{DL}$ & $>\mathrm{DL}$ & $>\mathrm{DL}$ & $>\mathrm{DL}$ & $>\mathrm{DL}$ & 0.005 & 0.022 & $>\mathrm{DL}$ & $>\mathrm{DL}$ \\
\hline A15 & $>\mathrm{DL}$ & $>\mathrm{DL}$ & $>\mathrm{DL}$ & 0.023 & $>\mathrm{DL}$ & $>\mathrm{DL}$ & 0.007 & 0.015 & $>\mathrm{DL}$ & $>\mathrm{DL}$ \\
\hline A16 & $>\mathrm{DL}$ & $>\mathrm{DL}$ & $>\mathrm{DL}$ & 0.005 & 0.019 & $>\mathrm{DL}$ & $0.009 *$ & $>\mathrm{DL}$ & 0.014 & $>\mathrm{DL}$ \\
\hline A17 & $>\mathrm{DL}$ & $>\mathrm{DL}$ & $>\mathrm{DL}$ & $>\mathrm{DL}$ & 0.019 & $>\mathrm{DL}$ & 0.008 & $>\mathrm{DL}$ & 0.022 & $>\mathrm{DL}$ \\
\hline A18 & $>\mathrm{DL}$ & $>\mathrm{DL}$ & $>\mathrm{DL}$ & $>\mathrm{DL}$ & 0.018 & $>\mathrm{DL}$ & 0.008 & $>\mathrm{DL}$ & 0.018 & $>\mathrm{DL}$ \\
\hline A19 & $>\mathrm{DL}$ & $0.015+$ & $0.167+$ & 0.031 & 0.028 & $0.090 *$ & $0.021+$ & $0.341 *$ & 0.020 & $0.064+$ \\
\hline A20 & $>\mathrm{DL}$ & $0.018+$ & $0.198+$ & 0.065 & 0.040 & $0.111^{*}$ & $0.024+$ & $0.493 *$ & 0.026 & $0.069+$ \\
\hline A 21 & $>\mathrm{DL}$ & $0.016+$ & $0.200+$ & 0.051 & 0.031 & $0.102 *$ & $0.019+$ & $0.699 *$ & $0.182 *$ & $0.069+$ \\
\hline A22 & $>\mathrm{DL}$ & $0.017+$ & $0.205+$ & 0.043 & 0.038 & $0.096 *$ & $0.022+$ & $0.850 *$ & 0.058 & $0.061+$ \\
\hline A 23 & $>\mathrm{DL}$ & $0.018+$ & $0.201+$ & 0.017 & 0.044 & $0.099 *$ & $0.021+$ & $0.359 *$ & 0.018 & $0.066+$ \\
\hline A24 & $>\mathrm{DL}$ & $0.019+$ & $0.226+$ & 0.122 & 0.046 & $0.092 *$ & $0.026+$ & $0.577 *$ & 0.069 & $0.061+$ \\
\hline A 25 & $>\mathrm{DL}$ & $0.016+$ & $0.134+$ & 0.021 & 0.018 & $0.075^{*}$ & $0.014+$ & $0.639 *$ & 0.009 & $0.040+$ \\
\hline A26 & $>\mathrm{DL}$ & $0.013+$ & $0.142+$ & 0.021 & 0.020 & $0.098^{*}$ & $0.015+$ & $0.398 *$ & 0.006 & $0.047+$ \\
\hline DL & 0.020 & 0.001 & 0.007 & 0.004 & 0.018 & 0.009 & 0.004 & 0.010 & 0.002 & 0.003 \\
\hline
\end{tabular}

DL = Detection Limit; $*$ Values above CONAMA 357/05 Class 1 to superficial waters; ${ }^{+}$Values above CONAMA 357/05 Class 3 to superficial waters. Legislative limits (Lgs. CONAMA 357/05 - Rating 1-3) of permitted metal amount in freshwater: Al 0.1-0.2 mg.L $\mathrm{L}^{-1}$; Cd 0.001-0.01 mg.L.

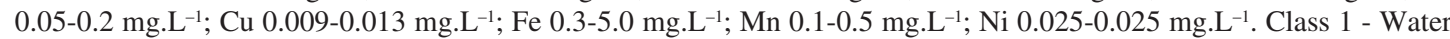
which may be intended for supply for human consumption after treatment simplified Class 3 - water which may be intended for supply for human consumption after conventional treatment or advanced. 
Table 5. Concentration of metals in the water during the dry season (mg. $\left.\mathrm{L}^{-1}\right)$.

\begin{tabular}{|c|c|c|c|c|c|c|c|c|c|c|}
\hline & Al & Cd & $\mathbf{P b}$ & Zn & $\mathrm{Cr}$ & Co & $\mathrm{Cu}$ & $\mathbf{F e}$ & Mn & $\mathbf{N i}$ \\
\hline $\mathrm{A} 1$ & $>\mathrm{DL}$ & 0.001 & $>\mathrm{DL}$ & 0.022 & $>\mathrm{DL}$ & 0.010 & $>\mathrm{DL}$ & $3.039 *$ & $0.144 *$ & 0.020 \\
\hline $\mathrm{A} 2$ & $>\mathrm{DL}$ & $0.002 *$ & $0.016^{*}$ & 0.014 & $>\mathrm{DL}$ & 0.013 & $>\mathrm{DL}$ & $0.347 *$ & $0.112 *$ & $0.028+$ \\
\hline $\mathrm{A} 3$ & $>\mathrm{DL}$ & $0.002 *$ & $>\mathrm{DL}$ & 0.027 & $>\mathrm{DL}$ & 0.015 & 0.004 & $0.503 *$ & $0.142 *$ & $0.028+$ \\
\hline $\mathrm{A} 4$ & $>\mathrm{DL}$ & $0.002 *$ & $0.013^{*}$ & 0.038 & $>\mathrm{DL}$ & 0.019 & 0.006 & 0.262 & $0.146^{*}$ & $0.030+$ \\
\hline A5 & $0.141^{*}$ & 0.001 & 0.010 & 0.075 & $>\mathrm{DL}$ & 0.011 & 0.005 & $0.421 *$ & 0.087 & 0.025 \\
\hline A6 & $0.160 *$ & $0.002 *$ & $0.036^{+}$ & $0.412 *$ & 0.028 & 0.028 & $0.017+$ & 0.283 & 0.098 & $0.036+$ \\
\hline A7 & $>\mathrm{DL}$ & 0.001 & 0.010 & 0.029 & 0.019 & 0.015 & $0.014+$ & 0.274 & 0.083 & $0.035+$ \\
\hline A8 & $0.206^{+}$ & $>\mathrm{DL}$ & $>\mathrm{DL}$ & 0.012 & $>\mathrm{DL}$ & $>\mathrm{DL}$ & $>\mathrm{DL}$ & $0.477^{*}$ & 0.043 & 0.009 \\
\hline A9 & $>\mathrm{DL}$ & $>\mathrm{DL}$ & $>\mathrm{DL}$ & 0.027 & 0.021 & 0.013 & 0.009 & $0.474^{*}$ & $0.104 *$ & $0.030+$ \\
\hline A 10 & 0.023 & $>\mathrm{DL}$ & $>\mathrm{DL}$ & 0.028 & 0.019 & 0.012 & 0.008 & $0.662 *$ & 0.100 & $0.034+$ \\
\hline A11 & $>\mathrm{DL}$ & $>\mathrm{DL}$ & $>\mathrm{DL}$ & 0.017 & $>\mathrm{DL}$ & $>\mathrm{DL}$ & $>\mathrm{DL}$ & 0.293 & 0.035 & 0.014 \\
\hline A12 & $>\mathrm{DL}$ & $0.002 *$ & $>\mathrm{DL}$ & 0.020 & 0.018 & 0.017 & 0.005 & $0.432 *$ & 0.049 & $0.032+$ \\
\hline A13 & $>\mathrm{DL}$ & 0.001 & $0.011^{*}$ & 0.013 & $>\mathrm{DL}$ & $>\mathrm{DL}$ & $>\mathrm{DL}$ & 0.089 & 0.006 & 0.021 \\
\hline A14 & $>\mathrm{DL}$ & 0.001 & 0.008 & 0.026 & 0.020 & 0.018 & 0.005 & $0.563^{*}$ & 0.023 & $0.034+$ \\
\hline A15 & $0.115^{*}$ & $>\mathrm{DL}$ & $0.011^{*}$ & 0.019 & $>\mathrm{DL}$ & $>\mathrm{DL}$ & $>\mathrm{DL}$ & 0.062 & 0.003 & 0.014 \\
\hline A16 & $>\mathrm{DL}$ & $>\mathrm{DL}$ & $0.012 *$ & 0.015 & $>\mathrm{DL}$ & 0.011 & 0.005 & 0.104 & 0.053 & 0.024 \\
\hline A17 & $0.178 *$ & $0.002 *$ & $0.015^{*}$ & 0.022 & 0.025 & 0.024 & 0.008 & 0.120 & 0.065 & $0.038+$ \\
\hline A18 & 0.035 & 0.001 & $0.011 *$ & 0.015 & 0.019 & 0.016 & 0.006 & 0.075 & 0.073 & $0.036+$ \\
\hline A19 & $>\mathrm{DL}$ & $>\mathrm{DL}$ & $0.021 *$ & $0.232 *$ & 0.031 & 0.016 & $0.029+$ & $0.449 *$ & 0.057 & $0.037+$ \\
\hline A 20 & $>\mathrm{DL}$ & $0.002 *$ & $0.017 *$ & 0.018 & $>\mathrm{DL}$ & 0.013 & 0.005 & 0.109 & 0.020 & $0.027+$ \\
\hline A 21 & $0.513^{+}$ & $0.005^{*}$ & $0.012^{*}$ & 0.025 & 0.026 & 0.023 & 0.008 & $1.019^{*}$ & $0.227 *$ & $0.038+$ \\
\hline A 22 & $>\mathrm{DL}$ & $0.004 *$ & $0.015^{*}$ & 0.033 & 0.029 & 0.020 & $0.012 *$ & $0.792 *$ & $0.122 *$ & $0.044+$ \\
\hline A23 & $0.429^{+}$ & $0.003 *$ & $>\mathrm{DL}$ & 0.013 & 0.024 & 0.019 & 0.007 & 0.090 & 0.034 & $0.032+$ \\
\hline A24 & $0.168 *$ & $0.003 *$ & $>\mathrm{DL}$ & 0.087 & 0.022 & 0.016 & 0.008 & $0.532 *$ & 0.082 & $0.032+$ \\
\hline A25 & $>\mathrm{DL}$ & $0.002 *$ & $0.012 *$ & 0.013 & $>\mathrm{DL}$ & 0.013 & $>\mathrm{DL}$ & $0.540 *$ & 0.025 & $0.026+$ \\
\hline A26 & $>\mathrm{DL}$ & 0.001 & $0.012 *$ & 0.013 & $>\mathrm{DL}$ & 0.013 & $>\mathrm{DL}$ & $0.348^{*}$ & 0.017 & $0.027+$ \\
\hline DL & 0.020 & 0.001 & 0.007 & 0.004 & 0.018 & 0.009 & 0.004 & 0.010 & 0.002 & 0.003 \\
\hline
\end{tabular}

DL = Detection Limit; *Values above CONAMA 357/05 Class 1 to superficial waters; ${ }^{+}$Values above CONAMA 357/05 Class 3 to superficial waters. Legislative limits (Lgs CONAMA 357/05 - Rating 1-3) of permitted metal amount in

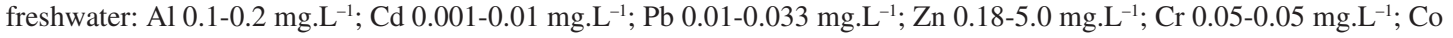
0.05-0.2 mg.L. ${ }^{-1}$; Cu 0.009-0.013 mg.L ${ }^{-1}$; Fe 0.3-5.0 mg.L - $^{-1}$; Mn 0.1-0.5 mg.L.-1; Ni 0.025-0.025 mg.L.- Class 1 - Water which may be intended for supply for human consumption after treatment simplified Class 3 - water which may be intended for supply for human consumption after conventional treatment or advanced.

found in Tables 6 and 7, together with the limits established by Brazilian law for metal (Lgs. CONAMA 344/04, 2004).

Brazilian legislation does not provide a sediment maximum value for the metals $\mathrm{Al}, \mathrm{Co}, \mathrm{Fe}$ and $\mathrm{Mn}$.

For Ni, the sites A1, A6, A7, A11, A14 and A20 in the dry season, showed concentrations above the level 1 . In the rainy season, site A9 showed higher concentration than allowed for level 1, and A5 showed higher concentration than the permissible amount for level 2 .

For $\mathrm{Cu}$, the sites A1, A2, A6, A7, A11, A14, A18 and A20 in the dry season, showed concentrations above the level 1 . In the rainy season, the sites A1, A8, A16 and A17 showed concentrations above level 1 .

For Cr, the sites A1, A2, A14 and A20 in the dry season, showed concentrations above the level 1 . Already in the rainy season, the sites A5 and A9 showed concentrations above level 1 .

For $\mathrm{Zn}$, only the point A20, in the dry season, has a concentration greater than that permitted for level 1 .

\subsection{Statistical analysis}

The Discriminant analysis (Figure 3) performed for the data base of surface water shows significant differences between the two metal collections, rainy and drought, with $\mathrm{p}=1.582 \mathrm{E}-21$. Sediment was not significantly different between the collections of drought and rainy, with $\mathrm{p}=0.7014$.

The PCA analysis (Figure 4) revealed greater sites characterisation for the rainy season by $\mathrm{Pb}, \mathrm{Cd}, \mathrm{Co}, \mathrm{Cu}$ and $\mathrm{Cr}$, while in the dry season, the analysis showed further characterisation by the metals $\mathrm{Al}, \mathrm{Mn}, \mathrm{Fe}$ and $\mathrm{Zn}$. 
Table 6. Concentration of metals in the sediment during the rainy season $\left(\mathrm{mg} \cdot \mathrm{kg}^{-1}\right)$.

\begin{tabular}{|c|c|c|c|c|c|c|c|c|c|c|}
\hline Sites & Al & $\mathrm{Cd}$ & $\mathbf{P b}$ & $\mathbf{Z n}$ & $\mathrm{Cr}$ & Co & $\mathrm{Cu}$ & $\mathrm{Fe}$ & Mn & $\mathrm{Ni}$ \\
\hline A1 & 11123.73 & 0.41 & 15.75 & 28.07 & 34.15 & 10.45 & $65.83^{*}$ & 1500.21 & 97.77 & 17.42 \\
\hline $\mathrm{A} 2$ & 12544.34 & $>\mathrm{DL}$ & 8.03 & 18.39 & 30.22 & 6.35 & 33.44 & 1467.13 & 101.69 & 10.29 \\
\hline A 3 & 2799.26 & $>\mathrm{DL}$ & $>\mathrm{DL}$ & 34.12 & 19.99 & $>\mathrm{DL}$ & 6.23 & 1349.95 & 24.89 & 4.29 \\
\hline A4 & 3277.34 & $>\mathrm{DL}$ & $>\mathrm{DL}$ & 24.83 & 24.68 & 2.43 & 8.06 & 1414.17 & 46.43 & 11.41 \\
\hline A5 & 7685.32 & $>\mathrm{DL}$ & 7.33 & 58.95 & $76.88^{*}$ & 5.29 & 22.22 & 1436.69 & 85.58 & $43.04+$ \\
\hline A6 & 3697.01 & $>\mathrm{DL}$ & 10.48 & 60.92 & 27.16 & 5.37 & 26.51 & 1448.33 & 94.31 & 7.62 \\
\hline A7 & 3505.16 & $>\mathrm{DL}$ & 6.42 & 63.24 & 16.49 & 2.90 & 20.46 & 1415.29 & 61.18 & 5.21 \\
\hline A 8 & 8011.93 & $>\mathrm{DL}$ & 5.44 & 28.66 & 26.87 & 4.18 & $35.98 *$ & 1423.29 & 57.68 & 14.75 \\
\hline A9 & 13568.65 & 0.32 & 24.10 & 54.25 & $43.25^{*}$ & 11.52 & 28.44 & 1457.93 & 90.45 & $18.80^{*}$ \\
\hline A10 & 6391.35 & 0.18 & 14.54 & 31.39 & 23.80 & 7.66 & 14.97 & 1376.05 & 43.19 & 12.62 \\
\hline A11 & 2456.39 & $>\mathrm{DL}$ & 8.61 & 9.58 & 19.72 & 5.52 & 7.45 & 1344.23 & 19.32 & 9.03 \\
\hline A12 & 9012.72 & 0.19 & 18.32 & 30.25 & 20.33 & 6.76 & 12.88 & 1386.00 & 48.27 & 9.48 \\
\hline A13 & 5755.85 & $>\mathrm{DL}$ & 12.86 & 13.32 & 22.33 & 6.32 & 6.95 & 1412.30 & 31.29 & 8.77 \\
\hline A14 & 6419.05 & $>\mathrm{DL}$ & 12.47 & 14.97 & 17.71 & 7.69 & 6.96 & 1423.04 & 40.02 & 9.10 \\
\hline A15 & 4148.20 & $>\mathrm{DL}$ & 10.86 & 11.97 & 12.60 & 5.19 & 6.88 & 1275.80 & $>\mathrm{DL}$ & 6.75 \\
\hline A16 & 4405.36 & $>\mathrm{DL}$ & 20.95 & 50.73 & 25.88 & 12.07 & $63.35 *$ & 1348.03 & 75.50 & 13.83 \\
\hline A17 & 12033.96 & 0.21 & 28.29 & 105.71 & 26.69 & 9.19 & $79.61 *$ & 1414.08 & 93.96 & 16.50 \\
\hline A18 & 2777.68 & $>\mathrm{DL}$ & 13.75 & 30.30 & 23.48 & 6.94 & 17.96 & 1342.07 & 38.19 & 10.05 \\
\hline A19 & 7585.45 & $>\mathrm{DL}$ & $>\mathrm{DL}$ & 41.65 & 17.02 & 3.93 & 15.31 & 1431.70 & 76.19 & 4.90 \\
\hline A20 & 6621.15 & $>\mathrm{DL}$ & $>\mathrm{DL}$ & 50.61 & 19.75 & 4.32 & 16.17 & 1431.81 & 50.68 & 5.33 \\
\hline $\mathrm{A} 21$ & 1176.90 & $>\mathrm{DL}$ & $>\mathrm{DL}$ & 10.80 & 2.96 & 2.20 & 7.41 & 1320.67 & 40.26 & $>\mathrm{DL}$ \\
\hline A22 & 1467.67 & $>\mathrm{DL}$ & $>\mathrm{DL}$ & 23.73 & 9.12 & 2.55 & 8.91 & 1361.38 & 39.07 & $>\mathrm{DL}$ \\
\hline $\mathrm{A} 23$ & 702.74 & $>\mathrm{DL}$ & $>\mathrm{DL}$ & 3.89 & 4.10 & $>\mathrm{DL}$ & 2.91 & 1193.02 & 43.14 & $>\mathrm{DL}$ \\
\hline A24 & 907.86 & $>\mathrm{DL}$ & $>\mathrm{DL}$ & 3.20 & 1.89 & $>\mathrm{DL}$ & 2.74 & 1041.83 & 10.94 & $>\mathrm{DL}$ \\
\hline A 25 & 2316.74 & $>\mathrm{DL}$ & $>\mathrm{DL}$ & 7.48 & 2.75 & 2.48 & 4.15 & 1361.04 & 71.52 & $>\mathrm{DL}$ \\
\hline A26 & 8956.23 & $>\mathrm{DL}$ & $>\mathrm{DL}$ & 6.45 & 0.83 & $>\mathrm{DL}$ & 2.34 & 10343.21 & 45.23 & $>\mathrm{DL}$ \\
\hline $\mathrm{DL}$ & 3.29 & 0.17 & 5.34 & 0.30 & 0.59 & 1.61 & 0.15 & 7.51 & 7.95 & 2.35 \\
\hline
\end{tabular}

DL = Detection Limit; *Values above CONAMA 344/04 Level 1 to sediments; ${ }^{+}$Values above CONAMA 344/04 Level 2 to sediments. Legislative limits (Lgs CONAMA 344/043- Rating 1-2) of permitted metal amount in sediment in freshwater:

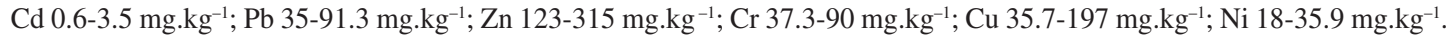
Level 1 - threshold below which provides a low probability of adverse effects to biota; Level 2 - threshold above which provides for a probable adverse effect on the biota.

\section{Discussion}

\subsection{Chemical parameters of water}

According to Calmano et al. (1993), the redox potential and $\mathrm{pH}$ affect the mobility of metals in sediment to the water column and the $\mathrm{pH}$ is the main mobilisation factor. Acid content of water and sediment in the environment lead to greater metal mobilisation (Chaillou et al., 2002). This mobility increases in oxygenated sediment. According to the data collected, despite high levels of oxygen, $\mathrm{pH}$ values did not promote mobilisation of metals in sediment to the water column. Therefore, the difference in concentrations of metals found in both periods and high levels of metals in water cannot be explained by the sediment-water metal mobilisation.

\subsection{Metals in surface water and sediment}

Sites A25 and A26, located on the Feijão river, show higher concentrations of $\mathrm{Cd}, \mathrm{Pb}, \mathrm{Cu}$ and $\mathrm{Ni}$ above the permitted level for Class 3 waters in the rainy season. This stream is responsible for supplying the municipalities of São Carlos and Itirapina - SP.

During the rainy season, the high contamination by cadmium, lead, copper and nickel can be related to the disposal of batteries in landfills located nearby bodies of water. These metals are considered "industrial metal", as they are found in low concentrations in nature, but used extensively in industrial processes (Järup, 2003).

Sites A19, A20 A21 and A22 located in downstream Monjolinho river, showed high levels of industrial metals $\mathrm{Pb}, \mathrm{Cd}, \mathrm{Ni}$ and $\mathrm{Cu}$. However, sites upstream A5, A8, A9 and A10 whose environment is occupied by large urban 
Table 7. Concentration of metals in the sediment during the dry season $\left(\mathrm{mg}^{\mathrm{kg}} \mathrm{kg}^{-1}\right)$.

\begin{tabular}{|c|c|c|c|c|c|c|c|c|c|c|}
\hline Sites & Al & Cd & $\mathbf{P b}$ & Zn & $\mathrm{Cr}$ & Co & $\mathbf{C u}$ & $\mathrm{Fe}$ & Mn & $\mathrm{Ni}$ \\
\hline A1 & 12103.70 & $>\mathrm{DL}$ & $>\mathrm{DL}$ & 34.81 & $46.95 *$ & 10.78 & $90.90 *$ & 2508.23 & 127.51 & $19.87 *$ \\
\hline $\mathrm{A} 2$ & 21598.26 & $>\mathrm{DL}$ & $>\mathrm{DL}$ & 22.37 & $43.39 *$ & 9.74 & $35.92 *$ & 2474.73 & 156.99 & 16.37 \\
\hline A3 & 6436.08 & $>\mathrm{DL}$ & $>\mathrm{DL}$ & 19.47 & 34.99 & 4.98 & 12.72 & 2330.92 & 52.47 & 9.51 \\
\hline A4 & 8439.46 & $>\mathrm{DL}$ & 7.36 & 63.23 & 30.21 & 7.02 & 18.70 & 2390.03 & 89.51 & 12.59 \\
\hline A5 & 9699.54 & $>\mathrm{DL}$ & 7.50 & 61.89 & 26.41 & 6.80 & 21.90 & 2343.18 & 60.19 & 11.09 \\
\hline A6 & 21432.26 & $>\mathrm{DL}$ & 13.46 & 110.97 & 29.19 & 12.68 & $47.58^{*}$ & 2489.99 & 134.75 & $22.45^{*}$ \\
\hline A7 & 22548.26 & $>\mathrm{DL}$ & 12.74 & 73.93 & 22.73 & 15.38 & $41.30^{*}$ & 2491.08 & 234.41 & $20.65^{*}$ \\
\hline A8 & 1710.36 & $>\mathrm{DL}$ & $>\mathrm{DL}$ & 3.81 & 15.43 & 5.34 & 5.62 & 2086.34 & 45.29 & 4.57 \\
\hline A9 & 8582.90 & $>\mathrm{DL}$ & 7.74 & 49.79 & 25.35 & 7.38 & 22.66 & 2234.50 & 29.67 & 10.91 \\
\hline A10 & 3721.94 & $>\mathrm{DL}$ & $>\mathrm{DL}$ & 20.25 & 27.97 & 6.40 & 15.08 & 2256.29 & 47.35 & 12.21 \\
\hline A11 & 28196.26 & $>\mathrm{DL}$ & 5.62 & 27.25 & 34.73 & 16.88 & $44.12 *$ & 2481.27 & 215.47 & $23.59 *$ \\
\hline A12 & 13027.36 & $>\mathrm{DL}$ & 20.02 & 23.85 & 28.67 & 9.48 & 14.86 & 2324.93 & 99.65 & 13.55 \\
\hline A13 & 3946.18 & $>\mathrm{DL}$ & $>\mathrm{DL}$ & 7.57 & 9.83 & 9.90 & 4.60 & 2260.92 & 275.53 & 10.05 \\
\hline A14 & 32728.26 & $>\mathrm{DL}$ & 26.58 & 63.57 & $53.61 *$ & 16.54 & $44.50 *$ & 2458.39 & 96.41 & $26.49 *$ \\
\hline A15 & 3536.96 & $>\mathrm{DL}$ & $>\mathrm{DL}$ & $>\mathrm{DL}$ & 14.09 & 7.32 & 3.88 & 1910.11 & 7.99 & 8.07 \\
\hline A16 & 1930.98 & $>\mathrm{DL}$ & $>\mathrm{DL}$ & 24.97 & 21.43 & 6.64 & 22.78 & 2202.37 & 37.95 & 10.35 \\
\hline A17 & 4495.58 & $>\mathrm{DL}$ & 8.22 & 65.43 & 21.03 & 6.78 & 33.54 & 2225.79 & 51.03 & 9.87 \\
\hline A18 & 4445.88 & $>\mathrm{DL}$ & 6.84 & 45.07 & 25.13 & 7.16 & $65.50 *$ & 2230.69 & 57.55 & 8.41 \\
\hline A19 & 3647.66 & $>\mathrm{DL}$ & $>\mathrm{DL}$ & 32.05 & 18.57 & 6.70 & 12.10 & 2208.36 & 30.35 & 8.35 \\
\hline A20 & 25508.26 & $>\mathrm{DL}$ & 15.72 & $190.65^{*}$ & $41.17 *$ & 17.50 & $55.38 *$ & 2454.58 & 175.03 & $25.79 *$ \\
\hline A21 & 5818.94 & $>\mathrm{DL}$ & $>\mathrm{DL}$ & 20.49 & 13.59 & 9.46 & 11.56 & 2208.90 & 72.37 & 8.43 \\
\hline A22 & 1252.60 & $>\mathrm{DL}$ & $>\mathrm{DL}$ & 17.97 & 15.79 & 5.90 & 7.72 & 2004.35 & 20.91 & 6.89 \\
\hline A23 & 2247.80 & $>\mathrm{DL}$ & $>\mathrm{DL}$ & $>\mathrm{DL}$ & 11.27 & 5.60 & 3.82 & 1964.86 & 28.27 & 5.15 \\
\hline A24 & 1485.28 & $>\mathrm{DL}$ & $>\mathrm{DL}$ & 4.97 & 12.25 & 4.86 & 5.22 & 1756.23 & 27.01 & 6.81 \\
\hline A 25 & 5903.20 & $>\mathrm{DL}$ & $>\mathrm{DL}$ & 13.17 & 6.09 & 5.32 & 5.98 & 2373.41 & 264.71 & 5.83 \\
\hline A26 & 1015.62 & $>\mathrm{DL}$ & $>\mathrm{DL}$ & 9.40 & 2.54 & 2.25 & 3.23 & 1305.11 & 73.65 & $>\mathrm{DL}$ \\
\hline DL & 3.29 & 0.17 & 5.34 & 0.30 & 0.59 & 1.61 & 0.15 & 7.51 & 7.95 & 2.35 \\
\hline
\end{tabular}

DL = Detection Limit; *Values above CONAMA 344/04 Level 1 to sediments; + Values above CONAMA 344/04 Level 2 to sediments. Legislative limits (Lgs CONAMA 344/043- Rating 1-2) of permitted metal amount in sediment in freshwater: Cd 0.6-3.5 mg.kg-1; Pb 35-91.3 mg.kg-1 Zn 123-315 mg.kg ${ }^{-1}$; Cr 37.3-90 mg.kg ${ }^{-1}$; Cu 35.7-197 mg.kg ${ }^{-1}$; Ni 18-35.9 mg. $\mathrm{kg}^{-1}$. Level 1 - threshold below which provides a low probability of adverse effects to biota; Level 2 - threshold above which provides for a probable adverse effect on the biota.
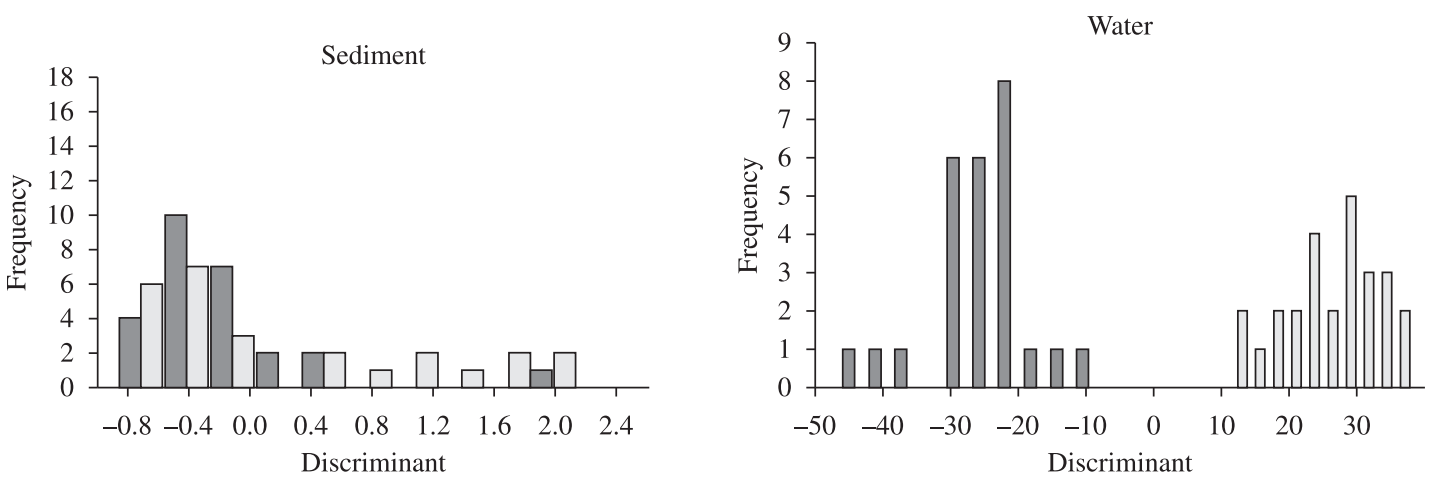

$\square$ Rainy season $\square$ Dry season

Figure 3. Discriminant analyses performed on water and sediment metals for rainy and dry season. 


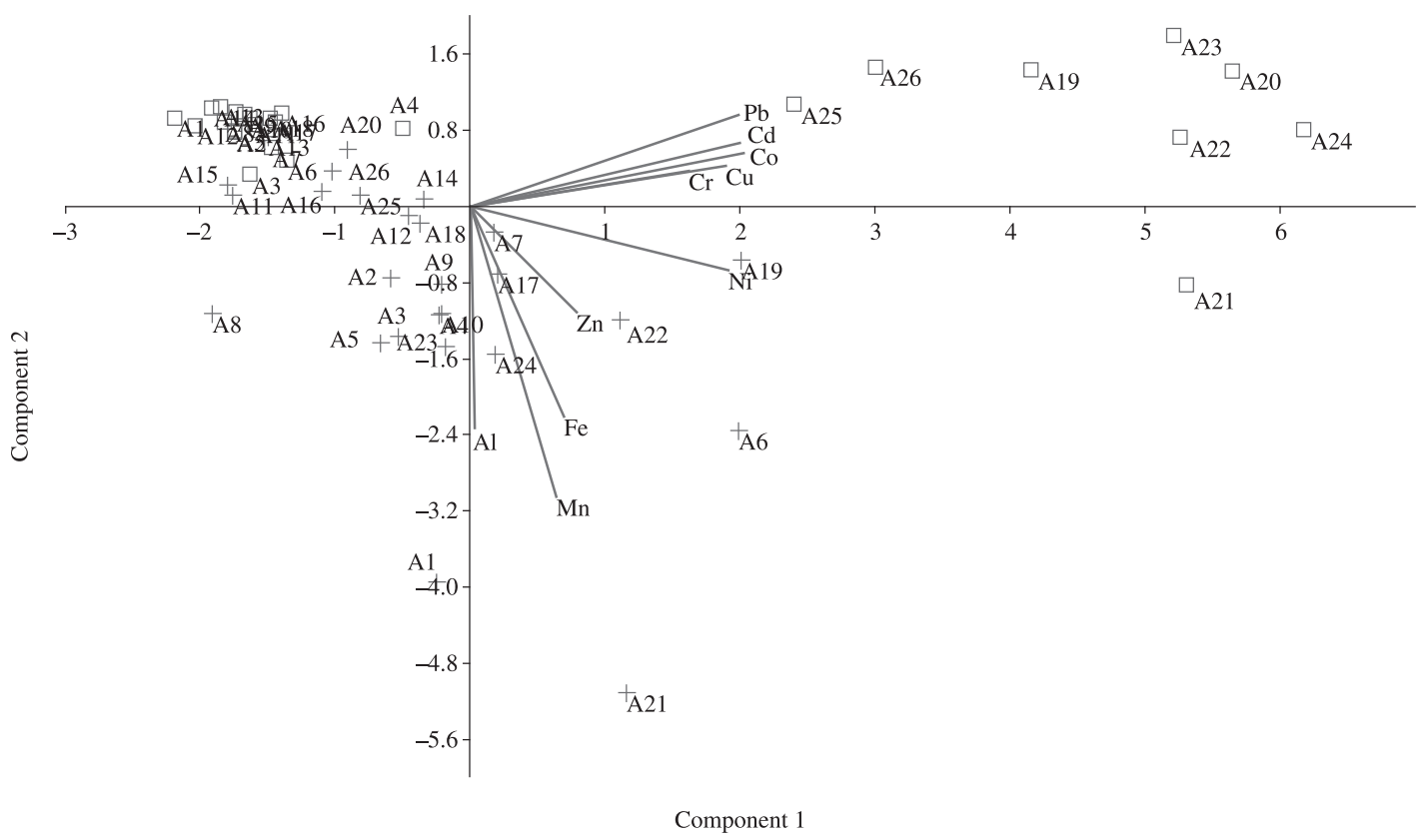

Figure 4. Principal Components Analysis for metals in water for the two collections $\square=$ Rainy; $+=$ Dry. Percentage of explanation $=69.28 \%$ for the first two axes.

density, did not show high levels of these metals. Thus the contamination of metals downstream Monjolinho river is not correlated with the presence of industries.

In the rainy season, there is a high leaching of the surrounding bodies of water. This causes the carrying of large quantities of particles and pollutants into rivers and streams, characterising the contribution of diffuse sources. Rietzler et al. (2001) related the elevated concentrations of metals recorded during the wet season to the landfill dump of the city and rain drainage. In addition, the riparian absence in streams is an aggravating factor for diffuse pollutants, as this vegetation is the main barrier to the uptake of leaching into water bodies.

São Carlos has a landfill located in the watershed the Feijão river. The landfill, in use between 1979 and 1996, has a high potential for contamination of groundwater, soil and surface water from its surroundings as it offers inadequate isolation of leached (Velozo, 2006). This landfill can be correlated with heavy metal contamination of industrial origin in the rainy season in sites of the Feijão river.

The city also has a dump still in use, located next to the Água Quente stream. This dump does not provide adequate isolation structure, characterising a high potential for contamination (Dimitriou et al., 2008) and may be correlated with high levels of heavy metals in the rainy season in sites A21, A23 and A24.

Kruskal-Wallis test between the concentrations of zinc at all sampling sites for periods of drought (average 0.0486) and rainy (average 0.0201), showed statistically significant differences $(p=0.01126)$. The highest concentration found in the dry season is probably due to fires to harvest cane sugar. High levels of zinc are found in this culture, both on the ground in plant biomass due to application of zinc sulfate ( $\mathrm{ZnSO} 4)$, widely used fertilizer. The fires, common practice in the dry season in the study area, produce ash with high concentrations of zinc, which reaches the water bodies (Ziolli, 1995).

Discriminant analysis showed no significant seasonal differences related to the metallic composition of the sediment during the study period. This is due to the stochastic nature of the sediment, where changes are perceived over a wider range of time. Therefore, sediment can act as an environmental indicator for large time intervals. By contrast, water has an ephemeral nature, since specific changes in space-time are reflected in its composition. Thus, the seasonal differences in the influence of metal behaviour on the environment are perceived in the water by the Discriminant analysis.

The metal punctual contamination (industrial waste, for example) is more evident in the dry season. This type of contamination provides a constant supply of metals in water. Thus, during rainy periods, the levels of metals in water bodies should be less than metal levels in periods of drought, due to decreased concentration of metals by high flows of rivers and streams. However, contamination by $\mathrm{Pb}, \mathrm{Cd}, \mathrm{Co}, \mathrm{Cu}$ and $\mathrm{Cr}$, from industrial processes occurred mainly in the rainy season, as demonstrated by the PCA.

Thus, this contamination may have originated from diffuse sources via leaching associated with landfills; because these landfills are close to the most impacted regions (Feijão river and Água Quente stream).

The origin of high concentrations of metals $\mathrm{Fe}, \mathrm{Al}$ and $\mathrm{Mn}$, present in the dry season, is from natural sources, since 
this metal composition is characteristic of the morphology of the savanna soil in this sub-basin (Queiroz-Neto, 1982).

\section{Conclusions}

São Carlos basin has high levels of industrial metal contamination in water bodies during the rainy season and $\mathrm{Zn}$ contamination in the water during the dry season. So, the levels and types of metals found in water are related to seasonality.

Contamination comes from diffuse sources. Thus, there was no evidence that there is disposal of industrial metal effluents directly into the water.

The high level of $\mathrm{Zn}$ found in the dry season is probably due to the burning of sugar cane, which release high levels of metal into the environment.

A diffuse source of contamination during the rainy season was probably due to products such as batteries, fluorescent lamps and chrome, whose presence in landfills can contaminate not only the soil but also aquifers and springs supply in the region. These landfills are located near sources of supply (Feijão river) and poorer neighbourhoods with high population density (Água Quente stream), presenting a potential risk of exposure for the population to these contaminated waters.

\section{References}

American Public Health Association - APHA, American Water Works Association - AWWA and Water Environment Federation - WEF, 2005. Standard methods for the examination of water and wastewater. 21nd ed. Washington: American Public Health Association. 874 p.

ANDERSEN, JM., 1976. An ignition method for determination of total phosphorus in lake sediments. Water Research, vol. 10, p. 329-331. http://dx.doi.org/10.1016/0043-1354(76)90175-5

ARMITAGE, PD., MOSS, D., WRIGTH, JF. and FURSE, MT., 1983. The performance of a new biological water quality score system based on macroinvertebrates over a wide range of unpolluted running-water sites. Water Research, vol. 17, p. 333347. http://dx.doi.org/10.1016/0043-1354(83)90188-4

BOUDOU, A. and RIBEYRE, F., 1989. Fundamental concepts in aquatic ecotoxicology. In BOUDOU, A. and RIBEYRE, F. (Eds.). Aquatic ecotoxicology: fundamental concepts and methodologies. CRC Press Inc. vol. 1, p. 35-75.

BOYD, RS., 2010. Heavy metal pollutants and chemical ecology: Exploring new frontiers. Journal of Chemical Ecology, vol. 36, p. 46-58. PMid:20108028. http://dx.doi.org/10.1007/s10886009-9730-5

BRAHIMPOUR, M. and MUSHRIFAH, I., 2008. Heavy metal concentrations in water and sediments in Tasik Chini, a freshwater lake, Malaysia. Environ Monitor Assess, vol. 141, p. 297-307. PMid:17891467. http://dx.doi.org/10.1007/s10661-007-9896-7

CALMANO, W., HONG, J. and FÖRSTNER, U., 1993. Binding and mobilization of heavy metals in contaminated sediments affected by $\mathrm{pH}$ and redox potential, vol. 28 (8-9), p.53-58.

CHAILLOU, G., ANSCHUTZ, P., LAVAUX, G., SCHÄFER, J. and BLANC, G., 2002. The distribution of Mo, U, and Cd in relation to major redox species in muddy sediments of the Bay of Biscay. Marine Chemistry, vol. 80, p. 41-59. http://dx.doi. org/10.1016/S0304-4203(02)00097-X

Conselho Nacional do Meio Ambiente - CONAMA, 2004. Resolução n ${ }^{\circ} 344$, de 25 de março de 2004. Estabelece as diretrizes gerais e os procedimentos mínimos para a avaliação do material a ser dragado em águas jurisdicionais brasileiras, e dá outras providências. Diário Oficial da República Federativa do Brasil. Brasília, DF, 07 maio 2004. Available from: <http://www.mma.gov. br/port/conama/res/res04/res34404.xml>. Access in: maio 2010.

-, 2005. Resolução n 357, de 17 de março de 2005. Dispõe sobre a classificação dos corpos de água e diretrizes ambientais para o seu enquadramento, bem como estabelece as condições e padrões de lançamento de efluentes, e dá outras providências. Diário Oficial da República Federativa do Brasil. Brasília, DF. 2005. Available from: <http://www.mma.gov.br/port/conama/res/res05/res35705. pdf $>$. Access in: maio 2010.

DIMITRIOU, E., KARAOUZAS, I., SARANTAKOS, K., ZACHARIAS, I., BOGDANOS, K. and DIAPOULIS, A., 2008. Groundwater risk assessment at a heavily industrialized catchment and the associated impacts on a peri-urban wetland. Journal of Environmental Management, vol. 88, p. 526-538. PMid:17499908. http://dx.doi.org/10.1016/j.jenvman.2007.03.019

DOI, H., TAKAGI, A. and KIKUCHI, E., 2007. Stream macroinvertebrate community affected by point-source metal pollution. International Review of Hydrobiology, vol. 92, no. 3, p. 258-266. http://dx.doi.org/10.1002/iroh.200610923

GOLDMAN, CR. and HORNE, AJ., 1983. Limnology. New York: McGraw-Hill Book Company. 464 p.

GOMES, LA., 1981. Aspectos qualitativos das águas pluviais urbanas. São Carlos: Escola de Engenharia de São Carlos, Universidade de São Paulo.105 p. Dissertação de Mestrado em Hidráulica.

Instituto de Pesquisa do Estado de São Paulo - IPT, 1995. Lixo municipal: Manual de gerenciamento integrado. São Paulo: IPT. 278 p. Publicação no. 2163

Instituto Nacional de Metrologia, Normalização e Qualidade INMETRO, 2009. Orientação sobre validação de métodos de ensaios químicos. Available from: <http://www.inmetro.gov. br/Sidoq/Arquivos/CGCRE/DOQ/DOQ-CGCRE-8_02.pdf>. Access in: maio 2010

JÄRUP, L., 2003. Hazards of heavy metal contamination. British Medical Bulletinvol, vol. 68, p. 167-182. PMid:14757716. http:// dx.doi.org/10.1093/bmb/ldg032

KIKUCHI, T., FURUICHI, T., HAI, HT., TANAKA, S., 2009. Assesment of heavy metal pollution in river water of Hanoi, Vietnam using multivariate analyses [J]. Bulletin of Environmental Contamination and Toxicology, vol. 8, no. 4, p. 575-582. http:// dx.doi.org/10.1007/s00128-009-9815-4

MASTOI, GM., SHAH, SGS. and KHUHAWAR, MY., 2008. Assessment of water quality of Manchar Lake in Sindh (Pakistan). Environmental Monitoring and Assessment, vol. 141, p. 287-296. http://dx.doi.org/10.1007/s10661-007-9895-8

MDEGELA, RH., BRAATHEN,M., REREKA, AEP., MOSHA, D., SANDVIK, M. and SKAARE, JU., 2009. Heavy metals and organochlorine residues in water, sediments, and fish in aquatic ecosystems in Urban and Peri-Urban areas in Tanzania. Water, Air, and Soil Pollution, vol. 203, p. 369-379. http://dx.doi.org/10.1007/ s11270-009-0019-7 
NIMER, E., 1972. Climatologia da Região Sudeste do Brasil: introdução à climatologia dinâmica. Revista Brasileira de Geografia, vol. 34, p. 3-48.

POVINELLI, J., 1972. Contribuição ao estudo da constante de desoxigenação da equação da DBO. São Carlos: Escola de Engenharia de São Carlos, Universidade de São Paulo.141 p. Tese de Doutorado em Engenharia Hidráulica e Saneamento.

PEDROSO, F., BONETTO, CA. and ZALOCAR, Y., 1988. A comparative study on phosphorus and nitrogen transport in the Parana, Paraguay and Bermejo rivers. In TUNDISI, JG. (Ed.). Limnologia e manejo de represas. São Carlos: Escola de Engenharia de São Carlos, Universidade de São Paulo. vol. 1, p. 91-117.

PELÁEZ-RODRÍGUEZ, M., 2001. Avaliação da qualidade da Bacia do Alto Jacaré-Guaçúl SP (Ribeirão do Feijão e Rio do Monjolinho) através de variáveis físicas, químicas e biológicas. São Carlos: Escola de Engenharia de São Carlos, Universidade de São Paulo. 147 p. Tese de Doutorado em Ciências da Engenharia Ambiental.

QUEIROZ-NETO JP., 1982. Solos da região dos cerrados e suas interpretações (revisão de literatura). Revista Brasileira de Ciência do Solo, vol. 6, p. 1-12.

RIETZLER, AC., FONSECA, AL., LOPES, GP., 2001. Heavy metals in tributaries of Pampulha Reservoir, Minas Gerais. Brazilian Journal of Biology, v. 61, n. 3, p. 363-370.

RIOS, L., 1993. Estudo limnológico dos córregos da água fria e da água quente. São Carlos: Universidade de São Paulo. 146 p. Dissertação de Mestrado em Ciências da Engenharia Ambiental.

SALAMI, LNBP., 1996. Estudo das influências climáticas e antropogênicas nas características físico-químicas do rio do Monjolinho, São Carlos, São Paulo. São Carlos: Escola de Engenharia de São Carlos, Universidade de São Paulo.135 p. Dissertação de Mestrado.

SANEI, H.,GOODARZI, F. and OUTRIDGE, PM., 2010. Spatial distribution of mercury and other trace elements in recent lake sediments from central Alberta, Canada: An assessment of the regional impact of coal-fired power plants. International Journal of Coal Geology, vol. 82, p. 105-115. http://dx.doi.org/10.1016/j. coal.2010.01.010

SANTOS, MJ., 1993. Estudo limnológico dos córregos da Água Fria e da Água Quente. São Carlos: Escola de Engenharia de São Carlos, Universidade de São Paulo. 291 p. Dissertação de Mestrado.
SANTOS, SAM., 1990. Educação ambiental através da analise de impactos de um rio (córrego do Gregório, município de São Carlos, SP). São Carlos: Centro de Divulgação Científica e Cultural. 27 p. Relatório Final do Projeto de Pesquisa.

SÉ, JAS., 1992. O rio do Monjolinho e sua bacia hidrográfica como integradores de sistemas ecológicos. São Carlos: Escola de Engenharia de São Carlos, Universidade de São Paulo. 381 p. Dissertação de Mestrado.

TEIXEIRA, D., 1993. Caracterização limnológica dos sistemas lóticos e variação temporal e espacial de invertebrados bentônicos na bacia do ribeirão do Feijão, São Carlos, SP. São Carlos: Universidade de São Paulo. Dissertação de Mestrado.

TEMPLETON, DM., ARIESE, F., CORNELIS, R., DANIELSSON, LG., MUNTAU, H., VAN LEEUWEN, HP. and LOBIÑSKI, R., 2000. Guidelines for terms related to chemical speciation and fractionation of elements. Definitions, structural aspects, and methodological approaches. Pure and Applied Chemistry, vol. 72, p. 1453-1470. http://dx.doi.org/10.1351/pac200072081453

TOLENTINO, M., 1967. Estudo crítico sobre o clima da região da São Carlos. São Carlos: Prefeitura Municipal. 78 p. Concurso de Monografias Municipais.

TUNDISI, JG., 2003. Água no século XXI: enfrentando a escassez. São Carlos: Rima Artes e Textos. vol. 1, 248 p.

United States Environmental Protection Agency - EPA, 1998. Method 3050 B. Available from: <http://www.epa.gov/SW-846/ pdfs/3050b.pdf>. Access in: jun. 2007.

VELOZO, R., 2006. Caracterização geológico-geotécnica do lixão desativado de São Carlos-SP, com o auxílio da geofísica. São Carlos: Escola de Engenharia de São Carlos, Universidade de São Paulo. Dissertação de Mestrado em Geotecnia.

ZIMMER, RK. and ZIMMER, CA., 2008. Dynamic scaling in chemical ecology. Journal of Chemical Ecology, vol. 34, p. 822836. http://dx.doi.org/10.1007/s10886-008-9486-3

ZIOLLI, RL., BARRETO, AS. and JARDIM, WF., 1995. Estudo preliminar da composição da água de chuva na região de Campinas - SP. In Anais da $18^{\text {nd }}$ Reunião anual da Sociedade Brasileira de Química, $7^{\text {nd }}$ encontro Brasileiro de fotoquímica e fotobiologia, 1995. Caxambu. 
